\title{
BMJ Open Associations of anthropometry since birth with sagittal posture at age 7 in a prospective birth cohort: the Generation XXI Study
}

\author{
Fábio A Araújo, , ${ }^{1,2}$ Raquel Lucas, , ,2 Andrew J Simpkin, ${ }^{3,4}$ Jon Heron,,4 \\ Nuno Alegrete, ${ }^{5,6}$ Kate Tilling, ${ }^{3,4}$ Laura D Howe, ${ }^{3,4}$ Henrique Barros ${ }^{1,2}$
}

To cite: Araújo FA, Lucas R, Simpkin AJ, et al. Associations of anthropometry since birth with sagittal posture at age 7 in a prospective birth cohort: the Generation XXI Study. BMJ Open 2017;7:e013412. doi:10.1136/ bmjopen-2016-013412

- Prepublication history and additional material for this paper are available online. To view these files please visit the journal online (http://dx.doi. org/10.1136/bmjopen-2016013412).

Received 9 July 2016 Revised 27 April 2017 Accepted 2 May 2017

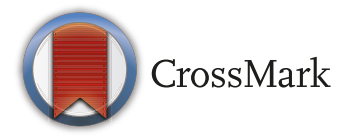

${ }^{1}$ ISPUP-EPIUnit, Universidade do Porto, Porto, Portugal

${ }^{2}$ Departamento de Ciências da Saúde Pública e Forenses e Educação Médica, Faculdade de Medicina, Universidade do Porto, Porto, Portugal

${ }^{3} \mathrm{MRC}$ Integrative Epidemiology Unit, Bristol, UK

${ }^{4}$ School of Social and

Community Medicine, University

of Bristol, Bristol, UK

${ }^{5}$ Centro Hospitalar São João,

Porto, Portugal

${ }^{6}$ Departamento de Cirurgia,

Faculdade de Medicina,

Universidade do Porto, Porto, Portugal

Correspondence to

Dr Fábio A Araújo;

fabio.araujo@ispup.up.pt

\section{ABSTRACT}

Objectives Adult sagittal posture is established during childhood and adolescence. A flattened or hypercurved spine is associated with poorer musculoskeletal health in adulthood. Although anthropometry from birth onwards is expected to be a key influence on sagittal posture design, this has never been assessed during childhood. Our aim was to estimate the association between body size throughout childhood with sagittal postural patterns at age 7.

Design Prospective cohort study.

Setting and participants A subsample of 1029 girls and 1101 boys taking part in the 7-year-old follow-up of the birth cohort Generation XXI (Porto, Portugal) was included. We assessed the associations between anthropometric measurements (weight, height and body mass index) at birth, 4 and 7 years of age and postural patterns at age 7 . Postural patterns were defined using latent profile analysis, a probabilistic model-based technique which allows for simultaneously including anthropometrics as predictors of latent profiles by means of logistic regression.

Results Postural patterns identified were sway, flat and "neutral to hyperlordotic"in girls, and "sway to neutral", flat and hyperlordotic in boys; with flat and hyperlordotic postures representing a straightened and a rounded spine, respectively. In both girls and boys, higher weight was associated with lower odds of a flat pattern compared with a sway/"sway to neutral"pattern, with stronger associations at older ages: for example, ORs were 0.68 ( $95 \% \mathrm{Cl} 0.53$ to 0.88 ) per SD increase in birth weight and $0.36(95 \% \mathrm{Cl} 0.19$ to 0.68$)$ per SD increase in weight at age 7 in girls, with similar findings in boys. Boys with higher ponderal index at birth were more frequently assigned to the hyperlordotic pattern ( $0 \mathrm{R}=1.44$ per SD; $\mathrm{p}=0.043$ ).

Conclusions Our findings support a prospective sculpting role of body size and therefore of load on musculoskeletal spinopelvic structures, with stronger associations as children get older.

\section{INTRODUCTION}

Sagittal standing posture evolves with growth and it contributes to the development of paediatric spinal deformities. ${ }^{1-3}$ Posture is

\section{Strengths and limitations of this study}

- This is the first study evaluating the role of anthropometric characteristics from birth through early childhood in shaping standing posture organisation in children.

- We assessed a large population-based cohort of 1029 girls and 1101 boys who were followed prospectively up to age 7-the Generation XXI study.

- Postural patterns were defined using a probabilistic, model-based method-latent profile analysiswhich included anthropometrics in addition to postural parameters.

- Although photogrammetry is the safest available method for postural evaluation of children, radiograms would have been the gold standard method for curvature measurement.

- Some degree of bias cannot be excluded, since children from the original cohort who were not included in this study were heavier and taller in the 4 and 7-year follow-up evaluations, and this could have changed the association between anthropometrics and postural patterns.

also crucial in the long term, ${ }^{4-8}$ since mature sagittal spinopelvic alignment is involved in a variety of orthopaedic disorders, ${ }^{2}$ such as degenerative disease and vertebral listhesis, as well as unspecific back pain and loss of function. ${ }^{2910}$

In the first months after birth, profound morphological changes to the pelvis and spine take place. ${ }^{458}$ There is an initial verticalisation of the pelvis, followed by the rising of the lordotic curve in the lower back as the child begins to assume a sustained upright position, leading the sacrum to a more horizontal position. ${ }^{4}$ Then, as walking abilities are acquired, constant dynamic adaptation takes place between pelvis shape, sagittal anatomy of the sacrum and physiological curves of the spine, all of which gradually develop and interact during growth. ${ }^{5}$ 
Children's anthropometry is expected to contribute to the mechanical framework of posture modulation, that is, weight and height modulate gravitational actions and regulate the net direction of forces imposed on the immature spinopelvic structures. ${ }^{11}$ Plastic deformation of bones, discs and other spinal structures can occur, ${ }^{12-14}$ as a result of reactive forces by muscles to ensure a stable centre of mass. ${ }^{468}$

Overall, there is strong biomechanical support for the hypothesis that children's anthropometric trajectories have the potential to shape postural morphotypes. However, this has never been empirically tested in a paediatric population. Longitudinal, population-based evidence is essential to assess the potential effects of body size in promoting a healthy posture, and also, to identify periods in childhood when prevention and management of weight disorders may be more effective for avoiding long-term musculoskeletal consequences of posture misalignment in later life.

By using prospective data from the Generation XXI birth cohort, our aim was to estimate the associations of body size from birth onwards with sagittal postural patterns at age 7 .

\section{METHODS}

This study is based on the population-based birth cohort Generation XXI, which has been previously described at length. ${ }^{15} 16$ Briefly, participants were recruited between 2005 and 2006 at five public maternity units serving the six municipalities of the metropolitan area of Porto, Portugal. At birth, 8647 infants were enrolled in the cohort (91.4\% of mothers invited agreed to participate). Four and 7 years after birth, $69 \%$ and $68 \%$, respectively, of all children recruited at birth were re-evaluated by face-to-face interviews and physical examinations. During the 7-year-old follow-up, a subsample of 2998 children consecutively assessed between December 2012 and August 2013, and without a diagnosis of severe neurological impairment, was invited for sagittal standing posture evaluation. Of those, $80 \%$ agreed to participate and attended the scheduled assessment. After excluding 118 girls and 165 boys with missing information on anthropometrics, 1029 girls and 1101 boys were included in the present analysis. The Generation XXI cohort study was approved by the Ethics Committee of São João Hospital/ University of Porto Medical School and complies with the Helsinki Declaration for medical research and with current national legislation, and was also approved by the National Committee of Data Protection. Written informed consent was obtained from all parents or legal guardians.

Birth weight and recumbent length at birth were retrieved from medical records by trained researchers. Ponderal index was then computed (weight in grams/ length in $\left.\mathrm{cm}^{3} \times 100\right) .{ }^{17}$ Additionally, weight and height were assessed at mean ages (SD) 4.3 (0.3) and $7.1(0.2)$ years. Weight was measured in light indoor clothing to the nearest $0.1 \mathrm{~kg}$ using a digital scale (TANITA) and height to the nearest $0.1 \mathrm{~cm}$ using a wall stadiometer (SECA). Body mass index (BMI) was defined as weight in kilograms divided by height in squared metres.

Sagittal standing posture evaluation in both genders occurred at 7.4 years of age on average (SD: 0.4 ), 0 to 420 days after the anthropometric evaluation $(50 \%$ of children evaluated within 61.5 days). Spherical retroreflective markers were placed over anatomical landmarks on the right side of the child's body: spinous processes of $\mathrm{C} 7$ and T12, anterior superior iliac spine, greater trochanter and lateral malleolus. Children were instructed to rest comfortably in habitual standing position with feet slightly apart, looking straight ahead and moving elbows forward. ${ }^{18} 19$ Floor markers were used to standardise children positioning. Full-body flash photographs of the sagittal right view of children were then acquired, after the examiner judged that the usual upright position had been attained. Angular measures formed by the lines drawn from the anatomical landmarks were obtained using the postural assessment software PAS $/ \mathrm{SAPO}^{20}$ : trunk, lumbar and sway angles (figure 1). These individual parameters were used to define postural morphotypes through the clustering algorithm Mclust, ${ }^{21}$ and a three-pattern solution was obtained separately for girls and boys. ${ }^{22}$ The geometric features (orientation, volume and shape) of the distributions of postural parameters were estimated from the data and allowed to vary between clusters or constrained to be the same for all the clusters. ${ }^{23}$ We then selected the type of model and number of clusters with the smallest Bayesian Information Criterion (BIC). ${ }^{24}$ This clustering procedure was chosen instead of the conventional heuristic methods ${ }^{13} 1925-27$ because it has the key advantage of allowing for testing different variances of angle measures within and across clusters.

In this paper, we replicated the three-pattern solution using the software Mplus V.6.12 (Muthén \& Muthén, Los Angeles, CA, USA), because the previously used clustering algorithm in the $\mathrm{R}$ package Mclust does not allow joint estimation of postural clusters and their associations with anthropometrics in the same model. This one-step approach was used to account for uncertainty in the assignment of patterns and consequently to obtain unbiased estimates of the association between anthropometrics and posture. ${ }^{28}$ Specifically, five latent profile models (different parametrisations of variance-covariance matrices) were tested in Mplus, with a fixed three-class solution for each gender. We selected the model with the highest concordance (observed agreement) for pattern assignment compared with the solution previously found in Mclust. ${ }^{22}$ Overall, concordance was $70 \%$ in girls and $78 \%$ in boys (detailed information provided in online supplementary table $\mathrm{S} 1$ ).

To quantify the associations of weight, height/length and body mass/ponderal index at birth, 4 and 7 years of age with postural patterns, we reran the selected models simultaneously using multinomial logistic regression (ie, including anthropometrics as predictors of postural 


\section{Trunk angle}

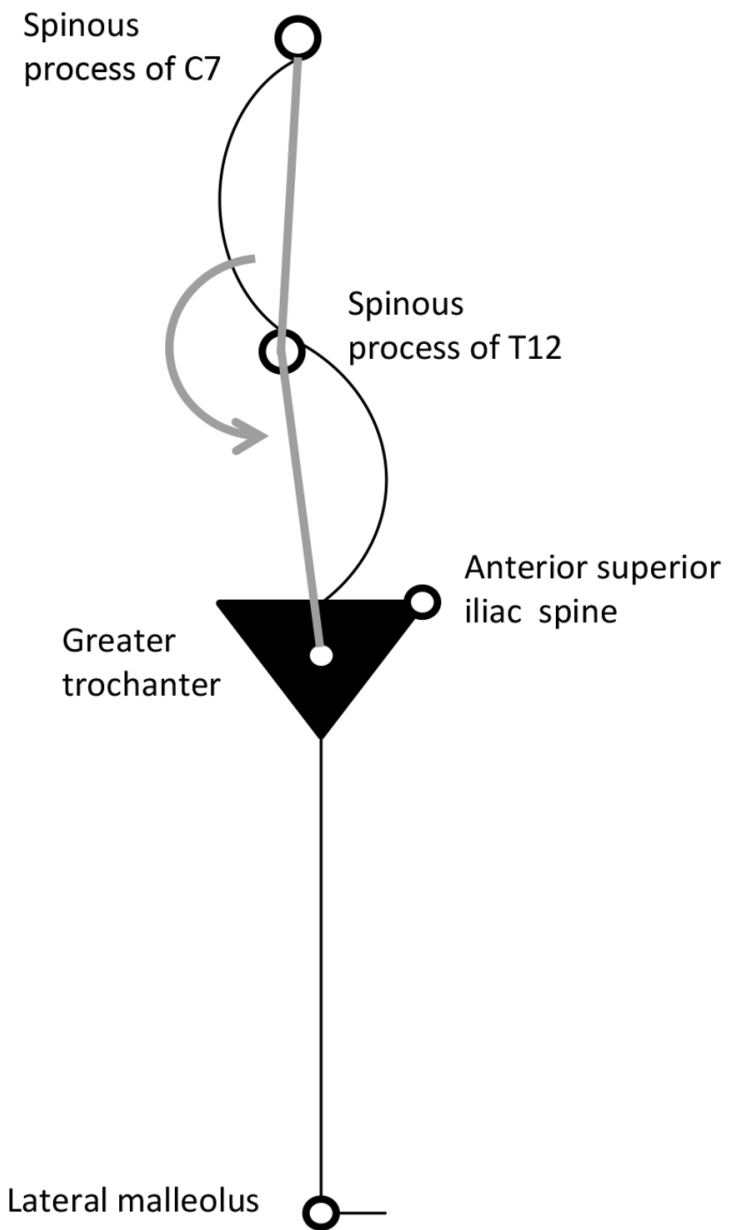

\section{Lower trunk inclination /}

Lumbar angle

Sway angle

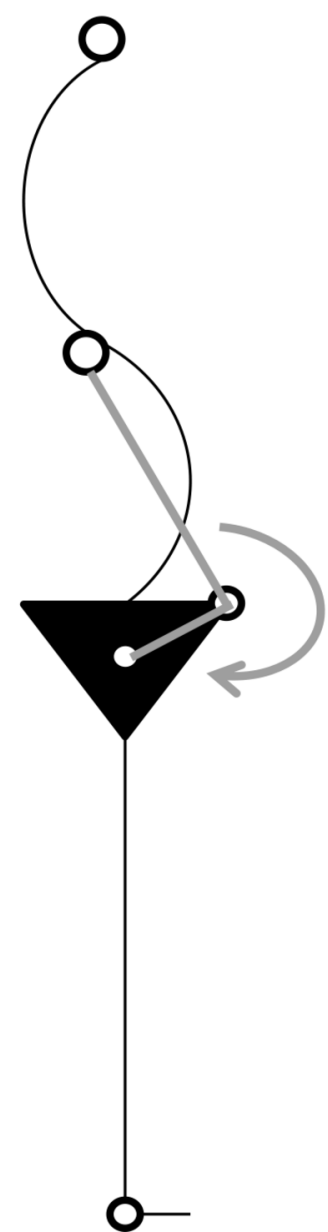

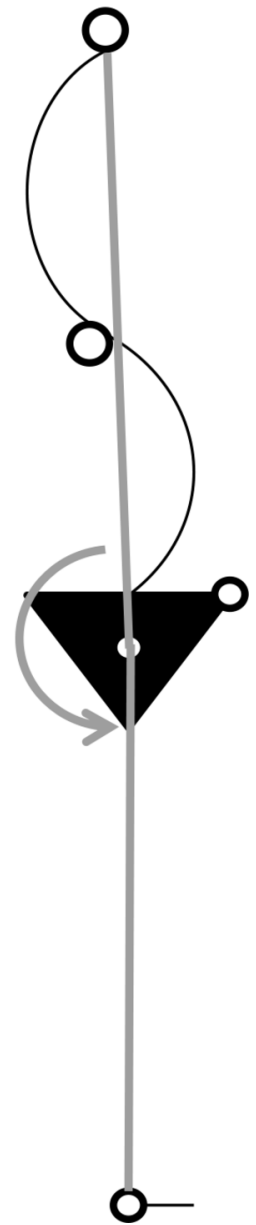

Figure 1 Individual angular measures used to identify sagittal postural patterns (using Mplus latent profile analysis).

latent profiles). Since the distributions of anthropometric variables change considerably during childhood, weight, height and BMI were standardised within each age through z-score transformations, by subtracting the mean value in the sample from each individual's value and dividing the result by the sample SD; units for associations are presented per SD. Estimates at age 4 were adjusted for birth measurements, and estimates at age 7 were adjusted for measurements at birth and 4 years; that is, weight estimates were adjusted for previous measurements of weight and similarly for height and BMI.

\section{RESULTS}

There was no association between inclusion in this study and anthropometric characteristics at birth. However, included girls and boys were lighter and shorter than those not at 4 and 7 years old (mean differences $(95 \%$ CI) ): $-0.29 \mathrm{~kg}(-0.53$ to -0.05$),-0.40 \mathrm{~kg}(-0.81$ to 0.01$)$, $-0.64 \mathrm{~cm}(-1.02$ to -0.26$)$ and $-0.42 \mathrm{~cm}(-0.83$ to -0.01$)$ in girls, and $-0.62 \mathrm{~kg}(-0.83$ to -0.40$),-0.81 \mathrm{~kg}(-1.20$ to
$-0.43),-0.84 \mathrm{~cm}(-1.21$ to -0.46$)$ and $-0.53 \mathrm{~cm}(-0.92$ to -0.13 ) in boys, respectively.

\section{Identification of postural patterns}

Individual angular measures were different between genders (multivariate analysis of variance, $\mathrm{p}<0.001$ ) with the main difference being higher lower trunk inclination/lumbar angle in girls $\left(4.90^{\circ}, \mathrm{p}<0.001\right)$.

In girls, the selected model was the one restricting variance of angular measures to be the same within patterns (identity covariance matrix) but allowing them to vary across patterns, while homogeneous variance was constrained only across patterns in boys (diagonal matrix). The average latent class probabilities (for the most likely latent class membership) varied between $0.73-0.81$ in girls and $0.72-0.86$ in boys. Figure 2 displays the features of the three postural patterns and angular values are provided in online supplementary table S2. The patterns were characterised by: increased trunk angle with backward tilt of the spine over the hips-decreased sway angle (sway in girls and 'sway to neutral' in boys given the high gender-specific prevalence of this pattern); straight 

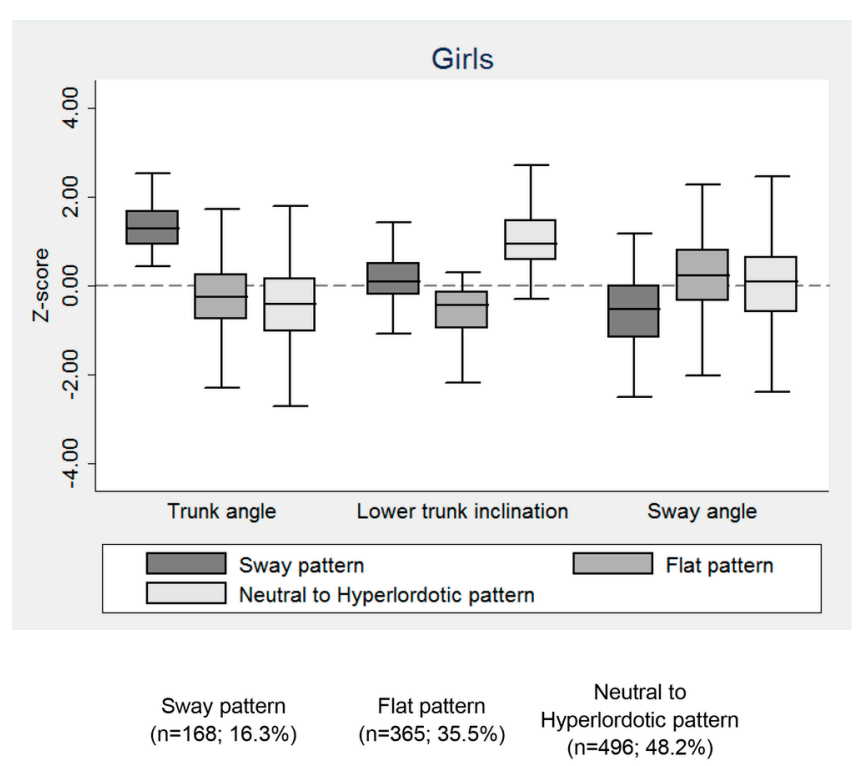
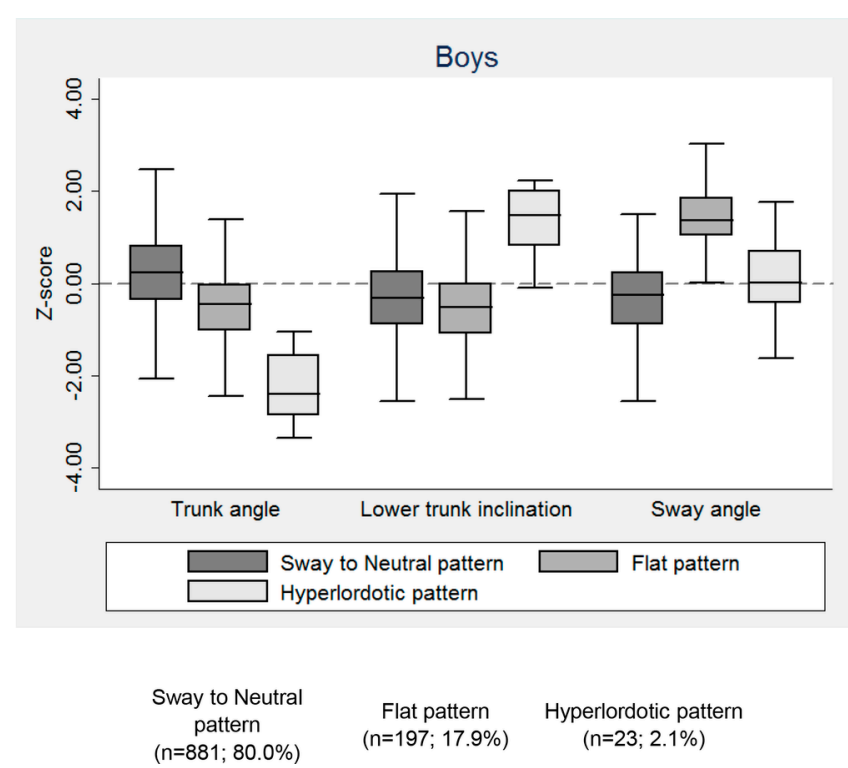

Figure 2 Box plots showing the distribution (median, IQR and range) of each separate postural angle, standardised to have a mean of zero and SD of one, across sagittal standing postural patterns, shown separately for girls and boys.

spine with forward trunk lean-increased sway angle (flat pattern in both genders); relatively increased lower trunk inclination and intermediate body sway ('neutral to hyperlordotic' pattern in girls) or extremely increased lower trunk inclination (hyperlordotic pattern in boys).

\section{Associations between anthropometry and sagittal posture}

Table 1 and figure 3 show descriptive analyses of the average anthropometric characteristics at birth and ages 4 and 7 years according to participants' most likely class assignment. ORs and respective 95\% CI for the associations between anthropometric traits and posture are shown in table 2, using the sway and 'sway to neutral' patterns as reference given their intermediate overall anthropometric profile.

\section{Girls}

Girls with the lowest average weight at all ages belonged more frequently to the flat pattern at age 7 . Higher weight at birth was associated with the sway pattern, while higher weight at ages 4 and 7 was related with a 'neutral to hyperlordotic' pattern. Per one SD increase in weight at birth, the odds of a flat pattern compared with sway changed by 0.68 (95\% CI 0.53 to 0.88 ). This association became stronger with age, with an OR of 0.36 (95\% CI 0.19 to 0.68 ) at age 7 . The same directions of associations were observed for body mass/ponderal index, with an OR of 0.68 (95\% CI 0.51 to 0.89 ) for flat compared with sway pattern per SD increase in birth weight and $0.39(95 \%$ CI 0.21 to 0.70 ) per SD increase in weight at 7 . Lower height was observed in children with the flat pattern, with mean (SD) height at age $7,122.45 \mathrm{~cm}$ (4.91) for the flat, $123.00 \mathrm{~cm}(5.25)$ for the 'neutral to hyperlordotic' and $123.06 \mathrm{~cm}(5.08)$ for the sway pattern. Taller girls were $22 \%$ to $40 \%$ less likely to develop a flat pattern at age 7 $(\mathrm{p} \leq 0.080)$, but these associations were weaker than those for weight/BMI.

\section{Boys}

As in girls, higher birth weight in boys was associated with the 'sway to neutral' pattern, while the highest weight thereafter was shown for those assigned to the hyperlordotic pattern. Per SD increase in weight, the OR for a flat pattern compared with sway/neutral was 0.66 at birth and 0.33 at 7 years old. The same decreasing trend for the flat type was observed in body mass/ponderal index with the OR being stronger than for weight. Boys who were born with higher ponderal index were more likely to have the hyperlordotic pattern ( $\mathrm{OR}=1.44$ per $0.27 \mathrm{~g} /$ $\left.\mathrm{cm}^{3} ; \mathrm{p}=0.043\right)$. Regarding length/height, boys showing a 'sway to neutral' pattern were born $0.97 \mathrm{~cm}$ longer, but those assigned to a hyperlordotic pattern reach a similar stature at 4 years old and were $1.04 \mathrm{~cm}$ taller at 7 years old, while shorter boys at birth were more likely to show a flat pattern $(\mathrm{OR}=0.65$ per $\mathrm{SD}(2.47 \mathrm{~cm}) ; \mathrm{p}=0.001)$.

\section{Sensitivity analysis}

Similar associations between anthropometry and sagittal posture were observed after restricting the sample to children assigned to the same postural pattern in both Mplus and Mclust (online supplementary table S3). Additionally, sensitivity analyses excluding twins (girls: $n=46$; boys: $\mathrm{n}=49$ ) and children born small or large for gestational age $^{29}$ (small: $\mathrm{n}=153$, large: $\mathrm{n}=45$ in girls; small: $\mathrm{n}=155$, large: $n=27$ in boys) and also including adjustment for gestational age at birth did not change the previous overall patterns of associations. Furthermore, socioeconomic conditions at birth were not clearly associated with postural patterns at 7 years old (maternal education: $\mathrm{p}=0.163$ in girls and $\mathrm{p}=0.074$ in boys; household income: $\mathrm{p}=0.436$ in girls and $\mathrm{p}=0.038$ in boys). Therefore, we opted not to include them as confounders of the relationships between anthropometrics and postural patterns. 
Table 1 Anthropometric characteristics at birth and ages 4 and 7 years according to sagittal standing postural patterns, shown separately for girls and boys

\begin{tabular}{|c|c|c|c|c|}
\hline & All & Sway pattern & Flat pattern & $\begin{array}{c}\text { Neutral to hyperlordotic } \\
\text { pattern }\end{array}$ \\
\hline & Mean (SD) & Mean (SD) & Mean (SD) & Mean (SD) \\
\hline \multicolumn{5}{|l|}{ Weight } \\
\hline Birth, $g$ & $3102.1(521.3)$ & $3155.7(507.5)$ & $3063.1(539.3)$ & $3112.5(511.2)$ \\
\hline 4 years, $\mathrm{kg}$ & $17.9(3.0)$ & $18.0(3.3)$ & $17.3(2.7)$ & $18.3(3.1)$ \\
\hline \multicolumn{5}{|l|}{ Length/height, cm } \\
\hline Birth & $48.2(2.3)$ & $48.4(2.1)$ & $48.2(2.5)$ & $48.2(2.3)$ \\
\hline 4 years & $104.3(4.5)$ & $104.6(4.5)$ & $104.1(4.4)$ & $104.4(4.6)$ \\
\hline 7 years & $122.8(5.1)$ & $123.1(5.1)$ & $122.4(4.9)$ & $123.0(5.3)$ \\
\hline \multirow{2}{*}{7 years, $\mathrm{kg} / \mathrm{m}^{2}$} & All & Sway to neutral pattern & Flat pattern & Hyperlordotic pattern \\
\hline & Mean (SD) & Mean (SD) & Mean (SD) & Mean (SD) \\
\hline \multicolumn{5}{|l|}{ Boys, $n=1101$} \\
\hline \multicolumn{5}{|l|}{ Weight } \\
\hline Birth, g & $3198.9(516.1)$ & $3229.3(491.0)$ & $3064.1(600.0)$ & $3189.3(519.0)$ \\
\hline 4 years, kg & $17.8(2.6)$ & $17.9(2.6)$ & $17.3(2.3)$ & $18.4(1.6)$ \\
\hline 7 years, $\mathrm{kg}$ & $25.8(4.7)$ & $26.0(4.8)$ & $24.5(3.9)$ & $26.9(4.1)$ \\
\hline \multicolumn{5}{|l|}{ Length/height, cm } \\
\hline 7 years, $\mathrm{kg} / \mathrm{m}^{2}$ & $16.70(2.19)$ & $16.85(2.24)$ & $16.00(1.79)$ & $17.19(1.98)$ \\
\hline
\end{tabular}

BMI, body mass index.

\section{DISCUSSION}

In this population-based birth cohort we analysed the associations of anthropometrics at different ages during childhood with sagittal posture at 7 years old. In both genders, children who remained lighter had an increased likelihood of a flat posture, and this relationship became stronger with increasing age. Concordantly, being heavier at 4 and 7 years old was associated with a posture characterised by increased lower trunk inclination/lumbar angle: 'neutral to hyperlordotic' in girls and hyperlordotic in boys. Shorter girls tended to present a flat posture and taller boys a hyperlordotic pattern.

This is the first study evaluating the role of anthropometric characteristics from birth and throughout childhood in shaping standing posture organisation. Our findings showed that adiposity was inversely related with a flattened spine, and concordantly, directly associated with a hyperlordotic posture. Only one other research group evaluated the relation between anthropometrics and patterns of standing posture before skeletal maturity is reached, ${ }^{1319}$ and cross-sectional analyses have shown that 14-year-old adolescents with a flat pattern had the lowest weight/BMI, while those in the hyperlordotic pattern were the fattest. ${ }^{19}$ Similarly, children in the flat pattern less frequently belonged to ascending, high or very high trajectories of body size defined from 3 to 14 years of age, while those in the hyperlordotic pattern were at higher risk of showing overweight trajectories. ${ }^{13}$

Classification systems of sagittal standing posture have been attempted in young adolescent girls ${ }^{26}$ and boys. ${ }^{25} 27$ However, different procedures for the definition of postural parameters preclude comparisons with our work. Particularly, children have been classified using a three-point Likert scale of uncorrected posture based 
Girls
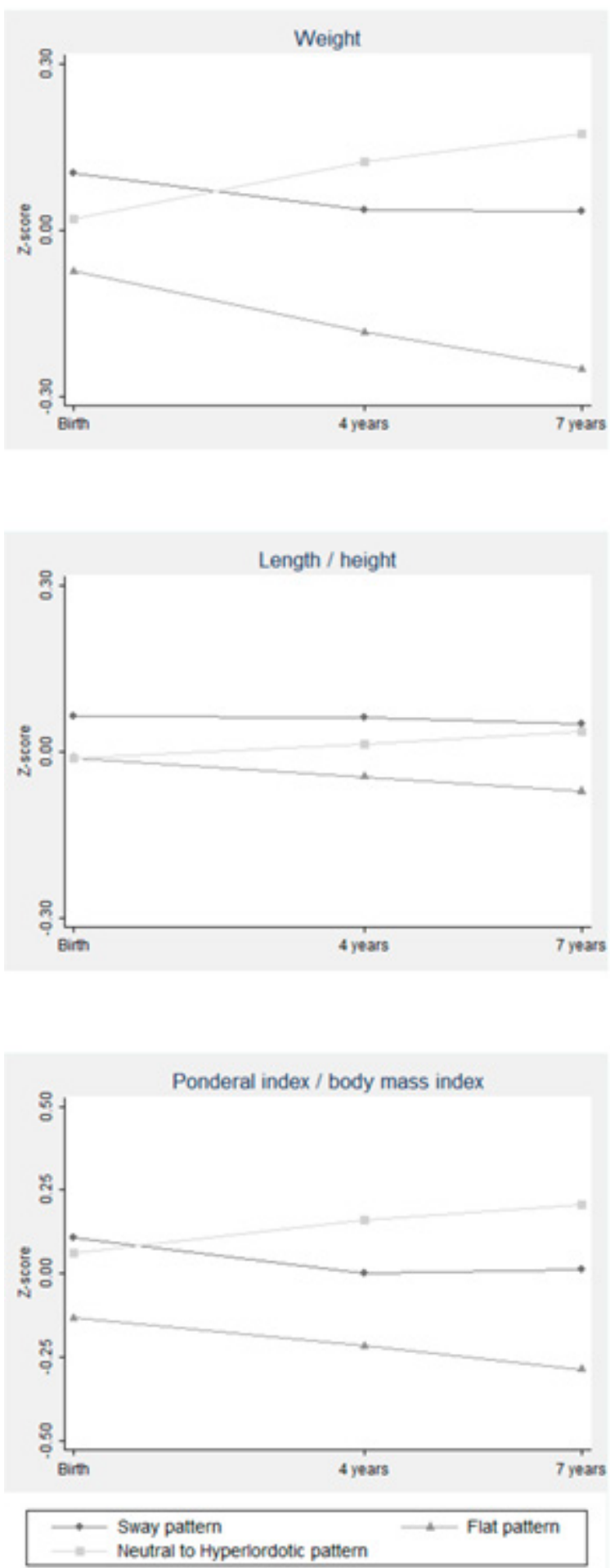

Boys
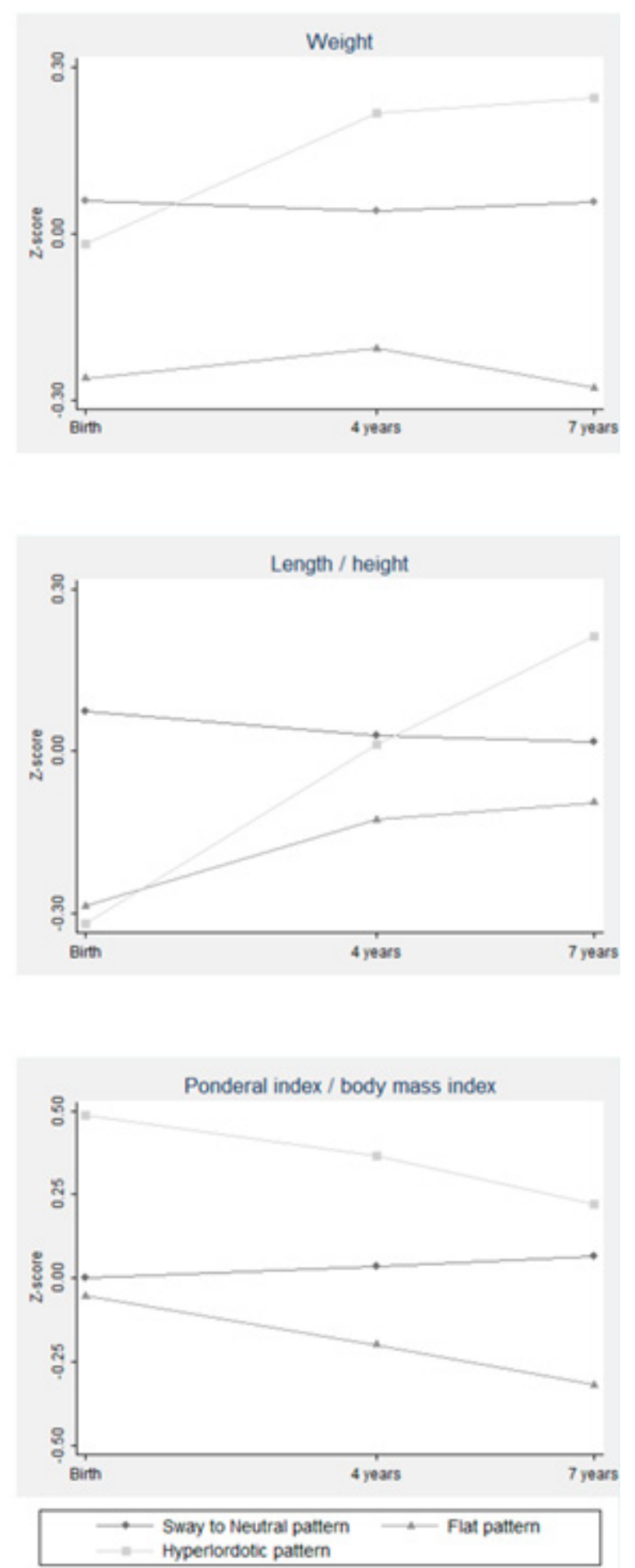

Figure 3 Standardised cross-sectional means of anthropometric characteristics at birth and ages 4 and 7 years across sagittal standing postural patterns, shown separately for girls and boys.

on the horizontal deviations of four body landmarks in respect to a vertical line ${ }^{27}$ and three global patterns (based on three angles with respect to the vertical) with different magnitude of spinal curves being observable within each pattern. ${ }^{25}{ }^{26}$ Our approach was to model postural patterns following three criteria: (1) to use postural parameters as continuous variables as a better representation of the natural spectrum of posture, (2) to search for variants comparable with those published in adolescents and adults, in a life course perspective and (3) to use a modelbased method that allowed for testing different variances of angle measures within and across clusters. Nevertheless, our patterns are comparable to those published by Dolphens $e t a l,{ }^{2526}$ at least regarding the suggested global 
Table 2 Associations between standardised anthropometric measures at birth, 4 and 7 years of age and sagittal postural patterns, shown separately for girls and boys

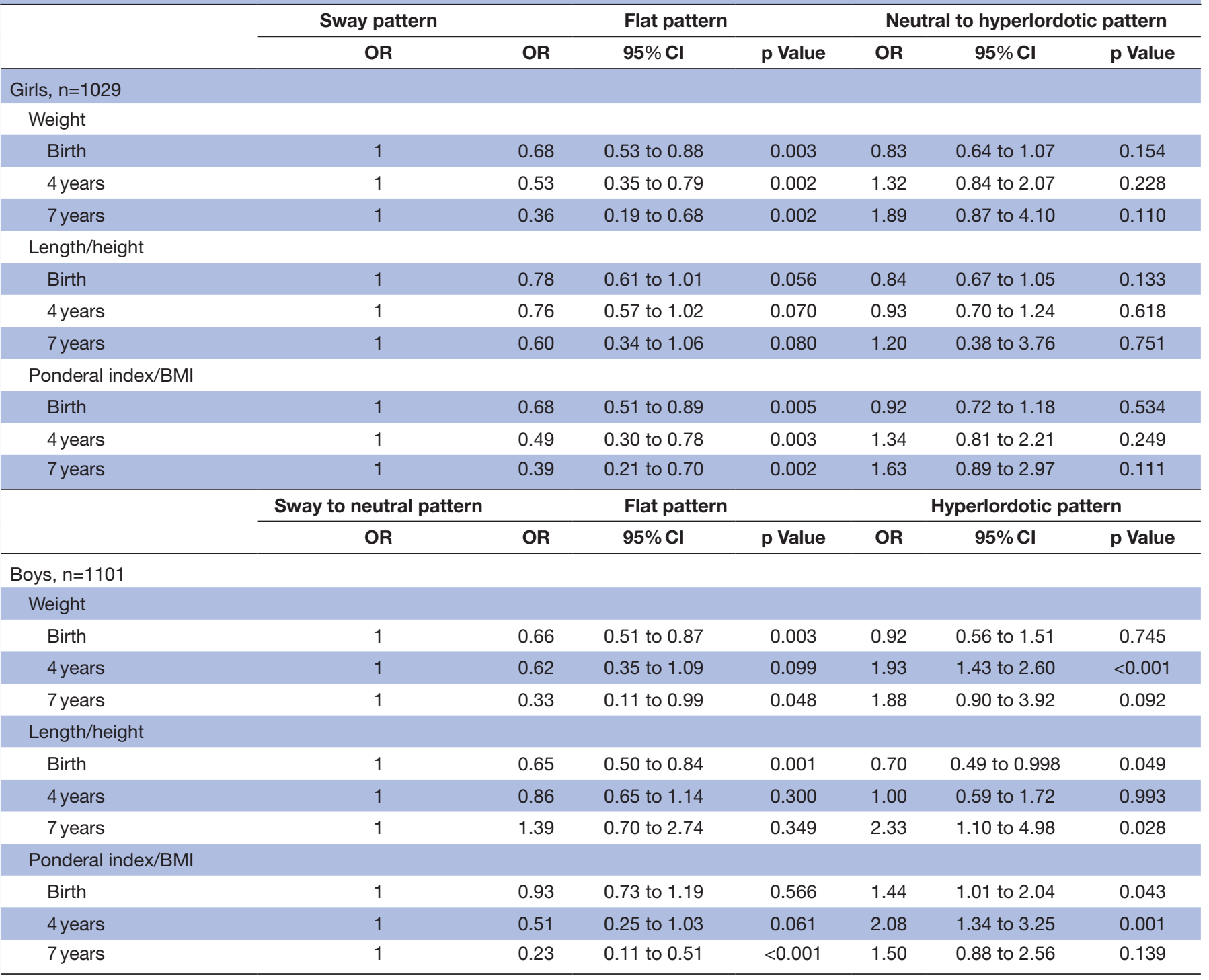

BMI, body mass index.

alignment classifications: neutral, sway back and leaning forward. In comparison to our classification and focusing on their features regarding the sway angle, those would, to some extent, correspond to the neutral, sway and flat patterns, respectively.

Our results during childhood are also consistent with cross-sectional findings in adult populations, ${ }^{12} 1430$ suggesting that higher adiposity levels during the development of posture is crucial for the shape and orientation of the spinopelvic unit, and implying a role of anthropometrics at early ages in shaping overall postural patterns during adulthood. In adults, both a flattened or hypercurved posture generally represent a poor postural health status on the basis of their relation with back pain ${ }^{31} 32$ and also by contributing to the aetiology of pattern-specific spine pathologies, such as discopathy and vertebral listhesis, respectively. ${ }^{11} 3334$ In contrast with later stages of life, ${ }^{1314193033}$ a neutral labelling was not considered appropriate to characterise postural patterns in children, but non-ideal patterns were already differentiated in childhood (flat and hyperlordotic), and it seems plausible that they will progressively mature and partially track over the life course. In terms of the clinical interpretation of our findings, we may postulate that low birth weight may contribute to a flat back that then may increase the risk of idiopathic scoliosis, ${ }^{1}$ while higher weight at 4 and 7 years of age may contribute to a hyperlordotic posture that then may predispose to Scheuermann's kyphosis. ${ }^{3}$

In both genders, anthropometric characteristics at ages 4 and 7 were more strongly associated with posture at 7 than body size at birth, as reflected by the age-related increase in the magnitude of associations between weight and the flat pattern, and also, the association of weight with increased lumbar curve observed only at ages 4 and 7 . Previous studies describing the changes 
in sagittal posture throughout different stages of growth, ${ }^{14-8}$ highlighted the potential effect of morphological anthropometric-related changes to be stronger during walking ages and potentially having a cumulative mechanical effect over time. Our observations at different ages in childhood support a cumulative result of weight bearing on spinopelvic structures, and that is the reason why our work focused on 7-year-old children. Although most changes in sagittal posture occur during the first months after birth, with the acquisition of upright position and walking abilities, ${ }^{458}$ it is important to allow sagittal curves to develop during growth through the influence of specific anthropometric mechanical environment and allow that different morphotypes could be distinguished within the sample. An additional major concern was to focus on prepubertal children to ensure homogeneity with regard to sexual development. ${ }^{35}$

Although height was associated with postural patterns, associations were generally weaker than for weight. Consequently, the latter seems to be the main driver of the direction and magnitude of the associations seen for BMI, as expected for a mechanical mechanism. However, this was not the case for the hyperlordotic pattern in boys, where height showed a stronger association with posture than weight. This particular relation between height/length and the hyperlordotic pattern may be a consequence of an anterior displacement of the centre of gravity related to an increased weight of the upper body as the child gets older. ${ }^{7}$ To re-establish a stable basis of support, the lumbar curve increases by means of higher vertebral growth, reflected in height, and this mechanism seems to be responsible for restoring sagittal balance. ${ }^{7}$ In agreement with this hypothesis, the hyperlordotic pattern was characterised by a substantially increased lower trunk inclination/lumbar angle. However, this study was not designed to investigate this pattern-specific association, and the usefulness of length/height to predict the presence of this particular pattern in school-aged boys deserves future specific exploration. Interestingly, the same average ascending trajectory of weight in the hyperlordotic pattern in boys was observed for the 'neutral to hyperlordotic' pattern in girls, even though with less extreme values of weight, which supports a functional aggregation of neutral and hyperlordotic postures in school-aged girls.

Some limitations of this work should be highlighted. Children from the original cohort not included in this study were heavier and taller during the 4 and 7 -year follow-up. Bias of our analysis is unlikely, as the association between anthropometry and postural patterns is unlikely to differ between included and excluded children. Although photogrammetry is the safest available method for postural evaluation of children, ${ }^{14} 1836$ radiographies directly allow us to measure spinal curvatures and are the gold standard which would have allowed more robust conclusions. Moreover, despite our efforts to standardise the position of the body and of the arms in particular, enough variability may have remained that could have influenced trunk position and therefore overall sagittal posture. Additionally, the present postural patterns have not yet been reproduced in other samples, and therefore future research is needed to confirm validation of the postural classifications. Furthermore, latent profile analysis in Mplus was performed in this study although Mclust $^{21}$ has been previously used for postural pattern identification. ${ }^{22}$ Since the two clustering methods use different estimation algorithms, ${ }^{37}$ classifications were not completely overlapping (online supplementary table S1). However, the solutions between the two clustering algorithms have been initially compared: while the same three-pattern solution was obtained in boys, for girls, Mplus suggested two and Mclust three patterns (based on the smallest BIC). Based on patterns' interpretability and also because Mplus solution aggregates two of the three groups suggested by Mclust, we opted to use three class models for both genders to replicate the solution provided by Mclust. ${ }^{22}$ Despite this, our conclusions should not be meaningfully affected since a good concordance between final models was obtained $(\geq 70 \%)$, as well as comparable face validity of patterns (ie, their postural meaning). Our findings were further supported by sensitivity analysis restricted to children assigned to the same postural pattern in both Mplus and Mclust, as shown in online supplementary table S3.

This is the first study evaluating the association of different measures of anthropometry and posture in children, using a large sample of children recruited from a population-based cohort with considerable variability both in exposure and outcome. Additionally, to examine overall postural patterns instead of isolated parameters is a key advantage because patterns allow a better characterisation of overall posture, permitting the analysis to account for the relationships between different anatomical regions. ${ }^{14} 1934$ Our work used, for the first time, a probability-based posture classification (ie, considering posterior probabilities of pattern membership) to avoid bias in the estimates of associations between anthropometry and postural patterns. ${ }^{28}$

We quantified the associations of early anthropometric features with sagittal posture during childhood, and we found that children who were lighter from the time of birth were more likely to develop a flattened posture at age 7 , while being heavier was associated with a hyperlordotic posture in both genders. The mechanical load imposed by body size seems to have a cumulative sculpting role throughout the first decade of life, especially after walking abilities are acquired.

Acknowledgements The authors gratefully acknowledge the families enrolled in Generation XXI and the contribution of the members of the research team and staff.

Contributors FAA, RL, NA and HB collaborated in the conceptualisation, design and acquisition of data. FAA, RL, AJS, JH, NA, KT, LDH and HB collaborated in the analysis and interpretation of data. FAA drafted the initial manuscript. RL, AJS, JH, NA, KT, LDH and HB critically reviewed the manuscript. All authors approved the 
final manuscript as submitted and agreed to be accountable for all aspects of the work.

Funding Generation XXI was funded by FEDER through the Operational Programme Competitiveness and Internationalization and by national funds through the FCT Fundação para a Ciência e a Tecnologia via grants POCI-01-0145-FEDER-016838 and POCl-01-0145-FEDER-016837, under the projects PTDC/DTP-EPI/1687/2014 and PTDC/DTP-EPI/3306/2014. Support by Administração Regional de Saúde Norte (Ministry of Health), Fundação Calouste Gulbenkian and Unidade de Investigação em Epidemiologia - Instituto de Saúde Pública da Universidade do Porto (EPIUnit) (POCl-01-0145-FEDER-006862; UID/DTP/04750/2013) is also acknowledged. Araújo FA and Lucas $\mathrm{R}$ were supported by grants SFRH/BD/85398/2012 and SFRH/ BPD/88729/2012, co-funded by FCT and the POPH/FSE.

Competing interests None declared.

Patient consent Obtained.

Ethics approval Ethics Committee of São João Hospital/University of Porto Medical School.

Provenance and peer review Not commissioned; externally peer reviewed.

Data sharing statement № additional data are available.

Open Access This is an Open Access article distributed in accordance with the Creative Commons Attribution Non Commercial (CC BY-NC 4.0) license, which permits others to distribute, remix, adapt, build upon this work non-commercially, and license their derivative works on different terms, provided the original work is properly cited and the use is non-commercial. See: http://creativecommons.org/ licenses/by-nc/4.0/

(c) Article author(s) (or their employer(s) unless otherwise stated in the text of the article) 2017. All rights reserved. No commercial use is permitted unless otherwise expressly granted.

\section{REFERENCES}

1. Schlösser TP, Vincken KL, Rogers K, et al. Natural sagittal spinopelvic alignment in boys and girls before, at and after the adolescent growth spurt. Eur Spine J 2015;24:1158-67.

2. Mehta VA, Amin A, Omeis I, et al. Implications of spinopelvic alignment for the spine surgeon. Neurosurgery 2012;70:707-21.

3. Jiang L, Qiu Y, Xu L, et al. Sagittal spinopelvic alignment in adolescents associated with Scheuermann's kyphosis: a comparison with normal population. Eur Spine J 2014;23:1420-6.

4. Mangione P, Gomez D, Senegas J. Study of the course of the incidence angle during growth. Eur Spine J 1997;6:163-7.

5. Marty C, Boisaubert B, Descamps $\mathrm{H}$, et al. The sagittal anatomy of the sacrum among young adults, infants, and spondylolisthesis patients. Eur Spine J 2002;11:119-25.

6. Cil A, Yazici M, Uzumcugil A, et al. The evolution of sagittal segmental alignment of the spine during childhood. Spine 2005;30:93-100.

7. Mac-Thiong JM, Berthonnaud E, Dimar JR, et al. Sagittal alignment of the spine and pelvis during growth. Spine 2004;29:1642-7.

8. Mac-Thiong J-M, Labelle $\mathrm{H}$, Roussouly $\mathrm{P}$, et al. Pediatric sagittal alignment. Eur Spine J 2011;20:586-90.

9. Lafage V, Schwab F, Patel A, et al. Pelvic tilt and truncal inclination: two key radiographic parameters in the setting of adults with spinal deformity. Spine 2009;34:E599-E606.

10. Kamitani K, Michikawa T, Iwasawa S, et al. Spinal posture in the sagittal plane is associated with future dependence in activities of daily living: a community-based cohort study of older adults in Japan. J Gerontol A Biol Sci Med Sci 2013;68:869-75.

11. Roussouly P, Pinheiro-Franco JL. Biomechanical analysis of the spino-pelvic organization and adaptation in pathology. Eur Spine $J$ 2011;20(Suppl 5):609-18.

12. Boulay C, Tardieu C, Hecquet J, et al. Sagittal alignment of spine and pelvis regulated by pelvic incidence: standard values and prediction of lordosis. Eur Spine J 2006;15:415-22.
13. Smith AJ, O'Sullivan PB, Beales DJ, et al. Trajectories of childhood body mass index are associated with adolescent sagittal standing posture. Int J Pediatr Obes 2011;6(2-2):e97-e106.

14. Araújo $F$, Lucas $R$. What do we know about the determinants of sagittal standing posture? OA Musculoskeletal Medicine 2014;2:15.

15. Correia S, Rodrigues $T$, Barros H. Socioeconomic variations in female fertility impairment: a study in a cohort of portuguese mothers. BMJ Open 2014;4:e003985.

16. Larsen PS, Kamper-Jørgensen M, Adamson A, et al. Pregnancy and birth cohort resources in Europe: a large opportunity for aetiological child health research. Paediatr Perinat Epidemiol 2013;27:393-414.

17. Davies DP. Size at birth and growth in the first year of life of babies who are overweight and underweight at birth. Proc Nutr Soc 1980;39:25-33.

18. Perry M, Smith A, Straker L, et al. Reliability of sagittal photographic spinal posture assessment in adolescents. Adv Physiother 2008;10:66-75.

19. Smith A, O'Sullivan P, Straker L. Classification of sagittal thoracolumbo-pelvic alignment of the adolescent spine in standing and its relationship to low back pain. Spine 2008;33:2101-7.

20. Ferreira EA, Duarte M, Maldonado EP, et al. Postural assessment software (PAS/SAPO): validation and reliabiliy. Clinics 2010;65:675-81.

21. Fraley C, Raftery AE. Model-Based clustering, discriminant analysis, and density estimation. J Am Stat Assoc 2002;97:611-31.

22. Araújo FA, Severo M, Alegrete N, et al. Defining patterns of sagittal standing posture in girls and boys of school age. Phys Ther 2017;97:258-67.

23. Fraley $\mathrm{C}$, Raftery $\mathrm{AE}$. Bayesian regularization for normal mixture estimation and model-based clustering. Journal of Classification 2007;24:155-81.

24. Schwarz G. Estimating the dimension of a Model. The Annals of Statistics 1978:6:461-4.

25. Dolphens $M$, Cagnie B, Coorevits $P$, et al. Classification system of the normal variation in sagittal standing plane alignment: a study among young adolescent boys. Spine 2013;38:E1003-E12.

26. Dolphens M, Cagnie B, Coorevits P, et al. Classification system of the sagittal standing alignment in young adolescent girls. Eur Spine $J$ 2014;23:216-25.

27. Pausić J, Dizdar D. Types of body posture and their characteristics in boys 10 to 13 years of age. Coll Antropol 2011;35:747-54.

28. Bray BC, Lanza ST, Tan X. Eliminating bias in classify-analyze approaches for latent class analysis. Struct Equ Modeling 2015;22:1-11.

29. Kramer MS, Platt RW, Wen SW, et al. A new and improved population-based canadian reference for birth weight for gestational age. Pediatrics 2001;108:E35.

30. Araújo F, Lucas R, Alegrete N, et al. Individual and contextual characteristics as determinants of sagittal standing posture: a population-based study of adults. Spine J 2014;14:2373-83.

31. Chaléat-Valayer E, Mac-Thiong JM, Paquet J, et al. Sagittal spinopelvic alignment in chronic low back pain. Eur Spine J 2011;20 Supp 5(Suppl 5):634-40.

32. Araújo F, Lucas R, Alegrete N, et al. Sagittal standing posture, back pain, and quality of life among adults from the general population: a sex-specific association. Spine 2014;39:E782-E794

33. Roussouly P, Gollogly S, Berthonnaud E, et al. Classification of the normal variation in the sagittal alignment of the human lumbar spine and pelvis in the standing position. Spine 2005;30:346-53.

34. Galbusera F, Brayda-Bruno M, Costa F, et al. Numerical evaluation of the correlation between the normal variation in the sagittal alignment of the lumbar spine and the spinal loads. J Orthop Res 2014;32:537-44

35. Patton GC, Viner R. Pubertal transitions in health. Lancet 2007:369:1130-9.

36. Fortin C, Feldman DE, Cheriet F, et al. Clinical methods for quantifying body segment posture: a literature review. Disabil Rehabil 2011;33:367-83

37. Haughton D, Legrand P, Woolford S. Review of three latent class cluster analysis packages: latent gold, poLCA, and MCLUST. Am Stat 2009;63:81-91. 